\title{
Universal Speed and Flux Estimator for Induction Motor
}

Mateusz Dybkowski

Wroclaw University of Science and Technology, Department of Electrical Machines,

Drives and Measurements

Received January 18, 2018; Accepted April 27, 2018

Abstract: In the paper, the concept of universal speed and flux estimator with additional parameters estimators is presented. Proposed solution is based on the Model Reference Adaptive System (MRAS) type flux and speed estimator and can be used in different industrial systems (especially in the automotive applications). Induction Motor (IM) parameters are estimated using the systems based only on simple simulators and adaptive systems (voltage model and current model). Proposed system was tested in the sensorless induction motor drive with the Direct Field Oriented Control (DFOC) algorithm. Simulation and experimental results are presented in the paper.

Keywords: Speed estimator • rotor flux estimator • parameter estimator • induction motor • MRAS • universal estimator • vector control

\section{Introduction}

The main advantage of sensorless induction motor drives are: less cabling, lower costs, good reliability and space minimization. Considering these reasons, different techniques for speed estimation were proposed (Tarchała et al., 2011) in the last two decades. All well-known speed estimators are characterized by large sensitivity to the IM parameter variations (Tarchała et al., 2011).

Due to these reasons, the sensorless drives have not found wide application as a basic system in industrial solutions. They can be used, for example, in the Fault Tolerant (FT) drives (Isermann, 2006; Dybkowski et al., 2014). The main problem of these systems is stable operation at low speed region and during field weakening operation (Tarchała et al., 2011).

Different methods of speed estimation, allowing for stable operation of the sensorless drives at speeds reaching several revolutions per minute at full load, have been presented in different papers. However, those methods are complicated, and they are not universal. These techniques are mainly based on Kalman Filter (KF) (Auger et al., 2013), Extended Kalman Filter (EKF) (Alonge et al., 2015; Auger et al., 2013), Unscented Kalman Filter (UKF) (Auger et al., 2013), Braided Kalman Filter (BKF) (Barut et al., 2008), Bi-input Kalman Filter (Barut, 2010; Barut et al., 2012), modified MRAS estimators (Dybkowski, 2013; Orlowska-Kowalska et al., 2014; Smith et al., 2016; Tarchała et al., 2011), extended state observer (Orlowska-Kowalska, 2003) or methods based on signal injection (Holtz, 2006).

The main problem in the speed reconstruction systems applied in the sensorless application is versatility (Dybkowski, 2013). Universal speed and flux estimators for AC drives should be stable in the whole speed reference changes (low and high-speed region), with and without the load torque changes. Furthermore, a universal estimator should reconstruct the real speed even in a very low speed region - including zero speed operation and during field weakening region. This estimator should work properly in different technological applications (Tarchała et al., 2011). 
Currently, the sensorless drives are developed as a post faulted application. They are dedicated for the Fault Tolerant Control (FTC) drives (Dybkowski et al., 2014), in particular for the drive resistant for the faults of rotor speed sensor or position sensor. Similar properties are required from these systems, like from the universal estimators. Additionally, simple construction and easy tuning of coefficients are required.

This paper describes the universal speed and flux estimator with additional IM parameter estimators for the speed sensorless induction motor drives (using DFOC method). Speed estimator is based on the MRAS ${ }^{C C}$ solution (Orlowska-Kowalska and Dybkowski, 2010). All simulation tests were performed in MATLAB Simulink software. Experimental tests were made on the laboratory set-up with DS1103 card.

\section{Concept of the Universal speed and flux estimator}

Different methods can be used for rotor speed and flux calculation. Simplified, the classification graph with different algorithmic techniques is presented in Fig. 1.

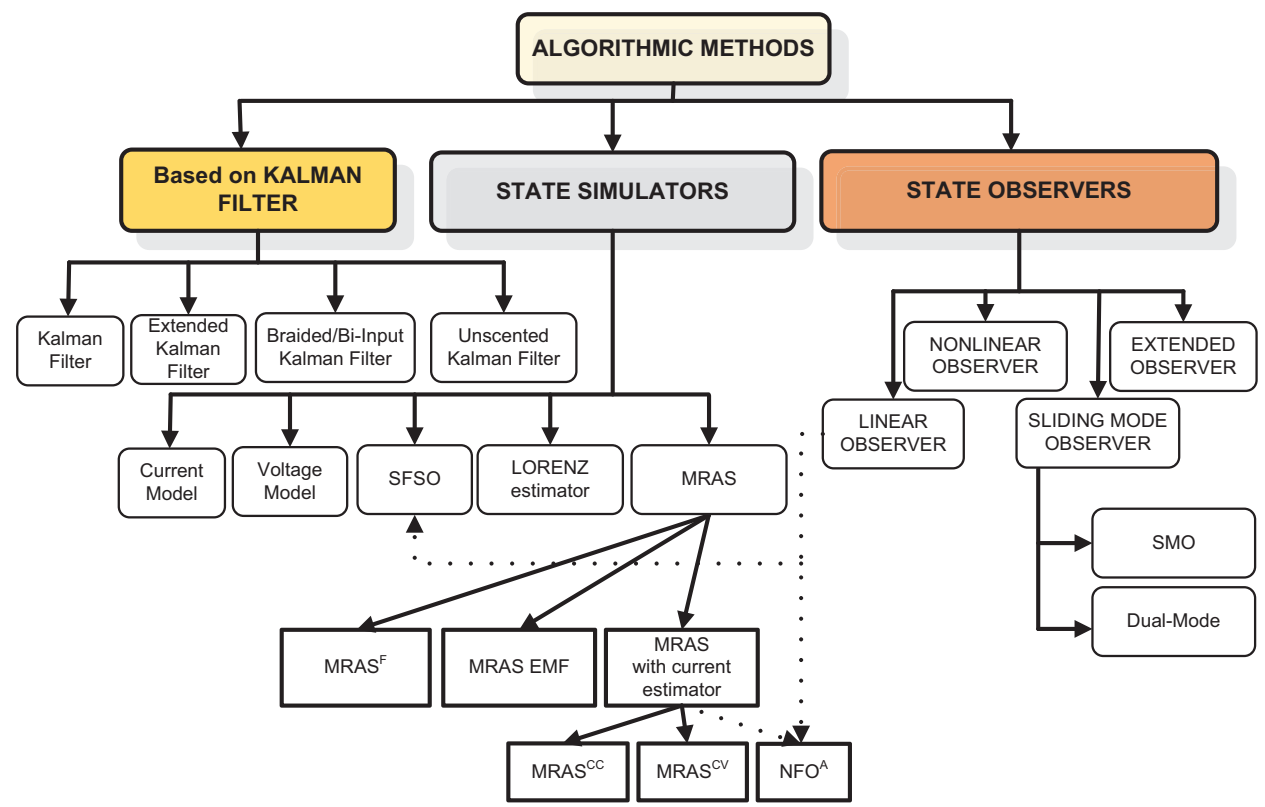

Fig. 1. Classification of induction motor state variables estimation methods (Tarchała et al., 2011)

Algorithmic methods, based on the mathematical model of the machine, depend on the induction motor (IM) parameters and thus are sensitive to motor parameter uncertainty and works stable only in a specified speed region (Rasmusen et al., 1999; Orlowska-Kowalska and Dybkowski, 2010; Tarchała et al., 2011). Those simple solutions, based on simple simulators, like voltage or current models, should be modified. One possible solution is based on expanding the vector of the estimated state variables with additional variables - parameters of the IM (Dybkowski, 2013).

In the paper, the novel speed and flux estimator (called universal) with additional parameter estimators is described.

\subsection{MRAS ${ }^{C C}$ estimator}

The universal speed and flux estimator is based on the well-known Model Reference Adaptive System (MRAS) with current model and current estimator, called MRAS ${ }^{\mathrm{cc}}$ (Orlowska-Kowalska and Dybkowski, 2010). This estimator is based directly on the mathematical model of the IM (Tarchała et al., 2011).

The rotor flux estimators, based on simple simulators - Voltage Model (VM) and Current Model (CM), can be expressed in $\alpha-\beta$ coordinates, in a per unit system [p. u.] by the equations (Tarchała et al., 2011):

$$
\frac{d}{d t} \boldsymbol{\psi}_{r}^{u}=\frac{x_{r}}{x_{m}}\left(\mathbf{u}_{\mathbf{s}}-r_{s} \mathbf{i}_{\mathbf{s}}-x_{s} \sigma \frac{d \mathbf{i}_{\mathbf{s}}}{d t}\right) \frac{1}{T_{N}},
$$




$$
\frac{d}{d t} \boldsymbol{\psi}_{r}^{i}=\left[\frac{r_{r}}{x_{r}}\left(x_{m} \mathbf{i}_{s}-\psi_{r}^{i}\right)+j \omega_{m}^{e} \psi_{r}^{i}\right] \frac{1}{T_{N}}
$$

where: $\Psi_{r}=\Psi_{r \alpha}+j \Psi_{r \beta} ; u_{s}=u_{s \alpha}+j u_{s \beta} ; i_{s}=i_{s \alpha}+j i_{s \beta}-$ rotor flux vector, stator voltage and current vectors, respectively, $r_{s}, r_{p}, x_{s}, x_{p}, x_{m}, x_{\sigma s^{\prime}}, x_{\sigma p}$ - stator and rotor resistances, stator and rotor reactance, magnetizing reactance, stator and rotor leakage reactance, $T_{N}=1 /\left(2 \pi f_{s N}\right), f_{s N}=50 H z, \sigma=1-x_{m}^{2} /\left(x_{s} x_{r}\right), \omega_{m}$ - motor speed.

Stator current estimator can be described by the equation (Orlowska-Kowalska and Dybkowski, 2010):

$$
\frac{d}{d t} \mathbf{i}_{s}^{e}=-\frac{r_{r} x_{m}^{2}+x_{r}^{2} r_{s}}{\sigma T_{N} x_{s} x_{r}^{2}} \mathbf{i}_{s}^{e}+\frac{1}{\sigma T_{N} x_{s}} \mathbf{u}_{s}+\frac{x_{m} r_{r}}{\sigma T_{N} x_{s} x_{r}^{2}} \Psi_{r}^{i}-j \omega_{m}^{e} \frac{x_{m}}{\sigma T_{N} x_{s} x_{r}} \Psi_{r}^{i}
$$

Stator current model (3) and rotor flux model (2) are adjusted by the estimated rotor speed (Orlowska-Kowalska and Dybkowski, 2010):

$$
\begin{aligned}
& \omega_{m}^{e}=K_{P}\left(e_{i_{s \alpha}} \Psi_{r \beta}^{i}-e_{i_{s \beta}} \Psi_{r \alpha}^{i}\right)+K_{I} \int\left(e_{i_{s \alpha}} \Psi_{r \beta}^{i}-e_{i_{s \beta}} \Psi_{r \alpha}^{i}\right) d t, \\
& e_{i_{s \alpha, \beta}}=i_{s \alpha, \beta}-i_{s \alpha, \beta}^{e} .
\end{aligned}
$$

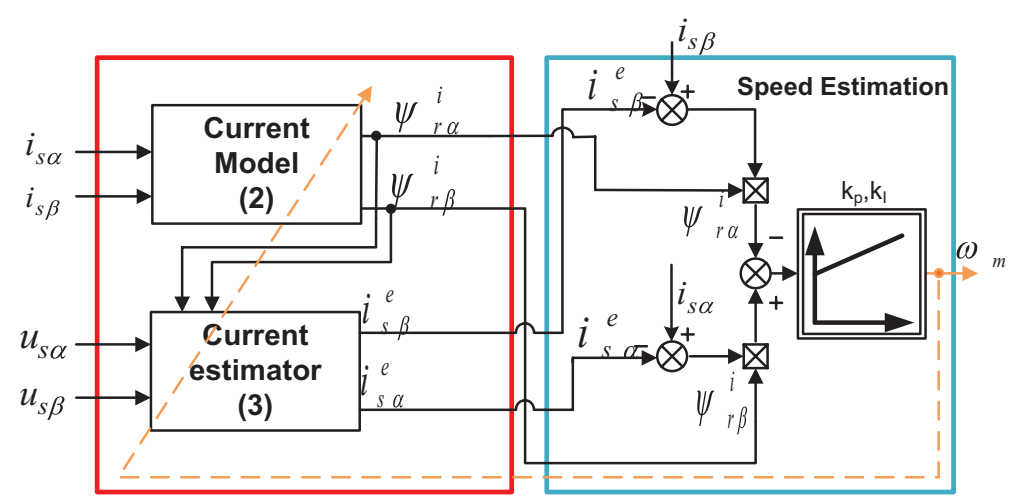

Fig. 2. Schematic diagram of the MRAS ${ }^{c c}$ speed estimator (Orlowska-Kowalska and Dybkowski, 2010; Tarchała et al., 2011)

Schematic diagram of this estimator is presented on Fig. 2.

\subsection{Magnetizing reactance estimator}

Parameters of IM are not constant - they depend on the temperature, current and motor speed (Orlowska-Kowalska et al., 2014). One of the most important parameter in the sensorless applications, especially in the traction drives, is a magnetizing reactance. This parameter is not constant, so the structure of the speed and flux estimator used in the sensorless drive should be modified by the estimated magnetizing reactance (Dybkowski and OrlowskaKowalska, 2010; Orlowska-Kowalska et al., 2014).

This parameter can be calculated from the equation (5) (in stator coordinate system) (Orlowska-Kowalska et al., 2014):

$$
x_{m}=\frac{\Psi_{m}}{i_{m}}
$$

where the magnetizing flux vector can be obtained from:

$$
\Psi_{m}=\sqrt{\Psi_{m \alpha}^{2}+\Psi_{m \beta}^{2}},
$$


and:

$$
\begin{aligned}
& \Psi_{m \alpha}=\int\left(u_{s \alpha}-r_{s} i_{s \alpha}\right) d t-x_{\sigma s} i_{s \alpha} \\
& \Psi_{m \beta}=\int\left(u_{s \beta}-r_{s} i_{s \beta}\right) d t-x_{\sigma s} i_{s \beta}
\end{aligned}
$$

The magnetizing current can be estimated using the known nonlinear inverse magnetizing curve:

$$
i_{m}=f\left(\Psi_{m}\right)
$$

Magnetizing curve can be represented in per unit [p. u.] as in (Levi et al., 2000):

$$
i_{m}=a \Psi_{m}+(1-a) \Psi_{m}^{b}
$$

where $a$ and $b$ are determined as constant coefficients characteristic for the given induction motor (Levi et al., 2000).

It was presented in (Levi et al., 2000) that proper choice of parameters a and $b$ guarantees the good reconstruction of this current versus magnetizing flux. In the Fig. 3, the magnetizing curves for different values of parameters a and $b$ are shown (Dybkowski and Orlowska-Kowalska, 2010).
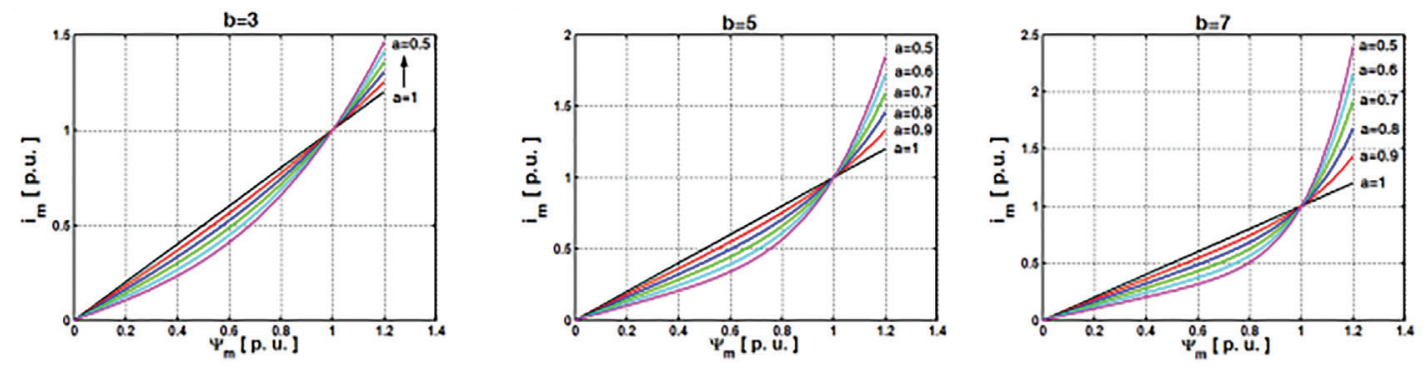

Fig. 3. The magnetizing curves of an IM for different parameters a and $b$ (Levi et al., 2000)

It was shown in the papers Orlowska-Kowalska et al. (2014) and Levi et al. (2000) that for low and medium power induction motors, parameter $b$ should be equal to 7. Parameter a should be set between 0.6 and 0.9 (Dybkowski and Orlowska-Kowalska, 2010).

\subsection{Stator resistance estimator}

The stator resistance changes significantly in the IM drives. Normally, it is caused by changes in drive operation conditions, temperature, humidity, and drive operation time. Changes of this parameter can reach up to $100 \%$ in difficult industrial conditions with limited cooling.

One of the simplest methods, commonly used to estimate the stator resistance, is the mechanism proposed in Vasić et al. (2003) and obtained by equation (10).

$$
r_{s}^{e}=\frac{u_{s}}{i_{s}}+\frac{T_{N} \frac{d}{d t}\left(i_{s}\left(x_{s} x_{r}-x_{m}^{2}\right)+\Psi_{r} x_{m}\right)}{i_{s} x_{r}},
$$

This mechanism ensures the correct estimation of this parameter in steady states and dynamic states. Due to the presence of a stator current derivative, this mechanism requires filtration of the estimated value with a relatively high time constant.

Simultaneous, parallel, estimation of the rotor speed and stator resistance (Vasić et al., 2003) is based on the adaptive system (Schauder, 1992). Such systems, despite the relatively extensive structure, are currently the most commonly developed group of methods for estimating stator resistance. This estimator is based on 
an MRAS concept with current and voltage models and resistance adaptation mechanism obtained by the equation (11):

$$
r_{s}^{e}=K_{\operatorname{Pr} s}\left(1+\frac{1}{s T_{I}}\right) e_{r s} ; \quad e_{r s}=i_{s \alpha}\left(\psi_{r \alpha}^{u}-\psi_{r \alpha}^{i}\right)+i_{s \beta}\left(\psi_{r \beta}^{u}-\psi_{r \beta}^{i}\right)
$$

In this concept, the current model is a reference system. The simplified diagram of the stator resistance and rotor speed estimator using MRAS ${ }^{\mathrm{CC}}$ estimator is shown in Fig. 4.

a)

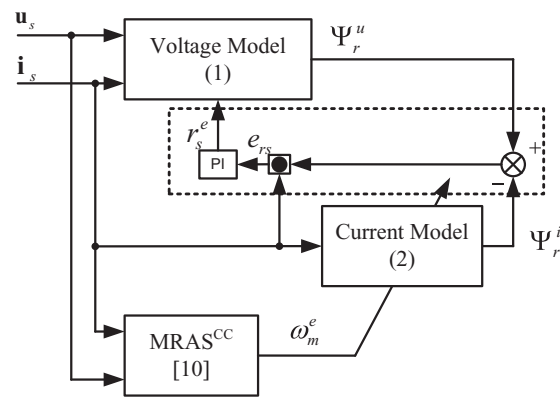

b)

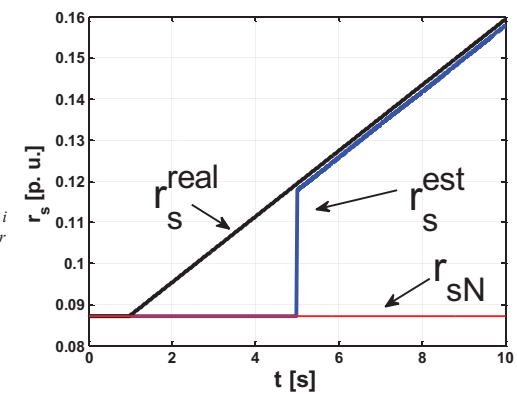

c)

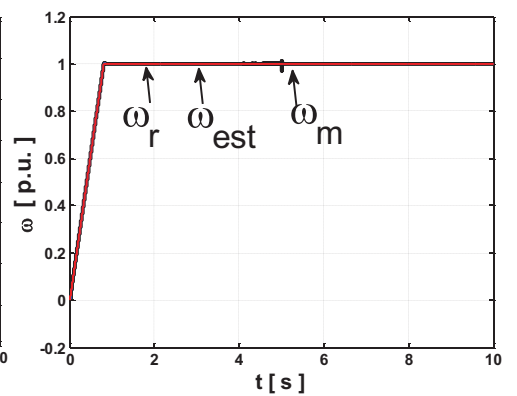

Fig. 4. Schematic diagram of parallel stator resistance estimator with the MRAS ${ }^{\mathrm{cc}}$ speed estimator (a), estimated stator resistance (b), estimated $\operatorname{speed}(\mathrm{c})$ for speed $\omega_{m}=\omega_{m N}$

It is possible to use a common current model in both algorithms. It is visible in Fig. 4 b) and Fig. 4 c) that stator resistance can be estimated correctly.

\subsection{Rotor resistance estimator}

The rotor resistance estimator proposed in (Zorgani et al., 2010) is also a solution based on the MRAS technique. Fig. 5 presents a simplified block diagram of the estimator. The construction resembles the classic MRASF solution (Schauder, 1992). In contrast to the speed estimator (based on MRAS idea), in this system, the angular velocity is a known quantity (estimated or measured).

Errors $\varepsilon_{\alpha}$ and $\varepsilon_{\beta}$ from equation (12) are used in the adaptation mechanism. Those signals depend on the difference between the two models.

$$
\left\{\begin{array}{l}
\varepsilon_{\alpha}=\psi_{r \alpha}^{u}-\psi_{r \alpha}^{i} \\
\varepsilon_{\beta}=\psi_{r \beta}^{u}-\psi_{r \beta}^{i}
\end{array}\right.
$$

a)

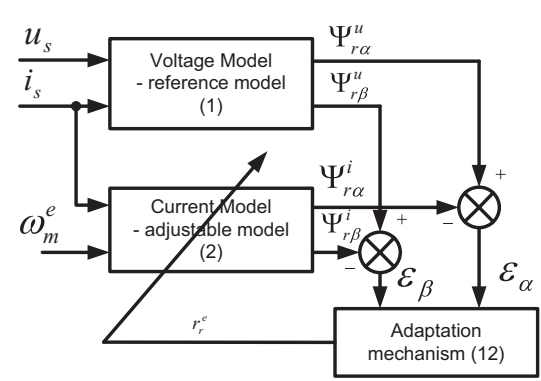

b)

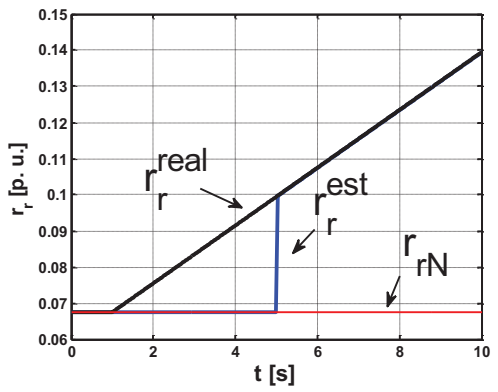

c)

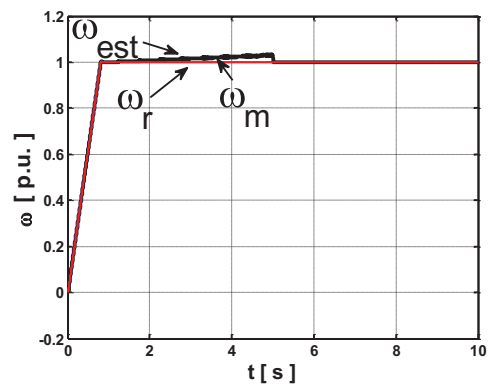

Fig. 5. Schematic diagram of the rotor resistance estimator (a), estimated rotor resistance (b), estimated speed (c) for speed $\omega_{m}=\omega_{m N}$ 
Adaptation mechanism can be obtained by:

$$
r_{r}^{e}=\frac{1}{s} A_{3}+A_{4} \text { where }\left\{\begin{array}{l}
A_{3}=K_{3}\left[\left(\frac{-\psi_{r \alpha}^{i}+x_{s} i_{\alpha s}}{x_{r}}\right) \varepsilon_{\alpha}+\left(\frac{-\psi_{r \beta}^{i}+x_{s} i_{\beta s}}{x_{r}}\right) \varepsilon_{\beta}\right] \\
A_{4}=K_{4}\left[\left(\frac{-\psi_{r \alpha}^{i}+x_{s} i_{\alpha s}}{x_{r}}\right) \varepsilon_{\alpha}+\left(\frac{-\psi_{r \beta}^{i}+x_{s} i_{\beta s}}{x_{r}}\right) \varepsilon_{\beta}\right]
\end{array} .\right.
$$

Parameters $K_{3}$ and $K_{4}$ - constant positive coefficients (Zorgani et al., 2010).

\subsection{Stator leakage reactance estimator}

The stator leakage reactance estimator, like previous estimators, is based on the adaptive system (Kubota et al., 1984). It consists of two tunable subsystems - the current model of the rotor flux and the stator current estimator. The stator current of the induction motor, which is treated as a reference system, is compared to the estimated current. The stator current estimator can be obtained by equations:

$$
\begin{aligned}
& \frac{d i_{s \alpha}^{e}}{d t}=-i_{s \alpha}^{e} \frac{r_{r} x_{m}^{2}+x_{r}^{2} r_{s}}{\sigma T_{N} x_{s} x_{r}^{2}}+\frac{1}{\sigma T_{N} x_{s}} u_{s \alpha}+\frac{x_{m} r_{r}}{\sigma T_{N} x_{s} x_{r}^{2}} \psi_{r \alpha}+\omega_{m} \psi_{r \beta} \frac{x_{m}}{\sigma T_{N} x_{s} x_{r}} \\
& \frac{d i_{s \beta}^{e}}{d t}=-i_{s \beta}^{e} \frac{r_{r} x_{m}^{2}+x_{r}^{2} r_{s}}{\sigma T_{N} x_{s} x_{r}^{2}}+\frac{1}{\sigma T_{N} x_{s}} u_{s \beta}+\frac{x_{m} r_{r}}{\sigma T_{N} x_{s} x_{r}^{2}} \psi_{r \beta}-\omega_{m} \psi_{r \alpha} \frac{x_{m}}{\sigma T_{N} x_{s} x_{r}} .
\end{aligned}
$$

This estimator, according to the above formula, depends on the stator current, stator voltage and the rotor speed. In these formulas, the stator reactance can be written as:

$$
x_{s}=x_{m}+x_{\sigma s}^{e}
$$

The adaptation mechanism uses the estimated and measured stator current. As a result of their comparison, an error is formed, described by the dependence:

$$
\begin{aligned}
& e_{i s \alpha}=i_{s \alpha}-i_{s \alpha}^{e} \\
& e_{i s \beta}=i_{s \beta}-i_{s \beta}^{e} .
\end{aligned}
$$

Leakage reactance can be calculated using the following algorithm:

$$
x_{\sigma s}^{e}=K_{P}\left(1+\frac{1}{s T_{I}}\right) e_{\psi}^{i \psi}, \quad e_{\psi}^{i \psi}=e_{i s \alpha} \psi_{r \beta}^{i}-e_{i s \beta} \psi_{r \alpha}^{i} .
$$

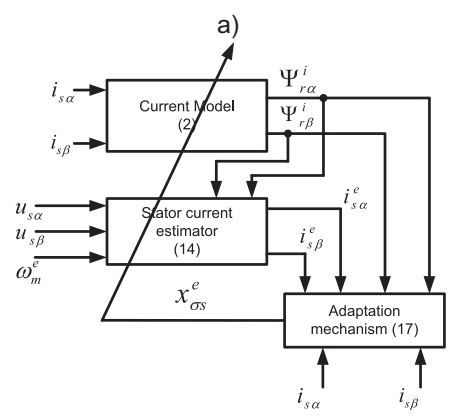

b)

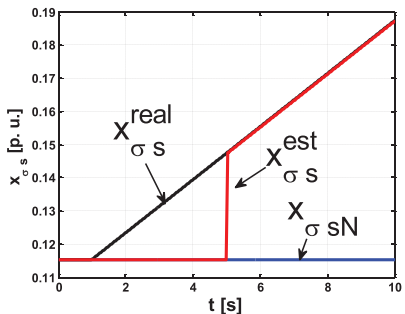

c)

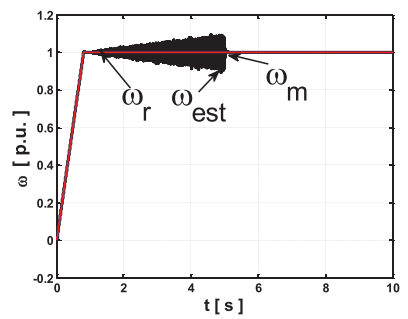

Fig. 6. Schematic idea of the leakage reactance estimator (a), estimated reactance (b), estimated speed (c) for speed $\omega_{m}=\omega_{m N}$

Fig. 6 presents a simplified block diagram of the estimator and estimation process for nominal speed. 


\subsection{Universal speed and flux estimator}

The universal estimator is a system that works stable for different drive conditions. This system is characterized by low sensitivity to the motor parameter changes. It is relatively simple in parameterization and can work properly in different control structures without having to change its settings. Such a system should guarantee correct operation of the drive for various types of induction motors without the parameter corrections (Dybkowski, 2013).

As a basis for the universal estimator, a system based on the MRAS concept was adopted - MRAS ${ }^{\text {cC }}$ (Dybkowski, 2013). To improve its operation in the low speed region, a modified mechanism for the rotor speed calculation was used (parameters are dependent on angular velocity). Also, additional systems for the estimation of induction motor parameters were added.

Application of a different speed estimation system (more sensitive to incorrect identification of the induction motor parameters) could lead to a loss of the drive stability in the case of incorrect estimation of the machine parameters by one of the additional estimators (e.g., dynamic states) (Dybkowski, 2013).

The schematic diagram of the universal estimator is shown in Fig. 7.

Induction motor parameter estimators, used in the proposed universal estimator, are based on the commonly known simple estimators and can be combined into one estimator based on these models.

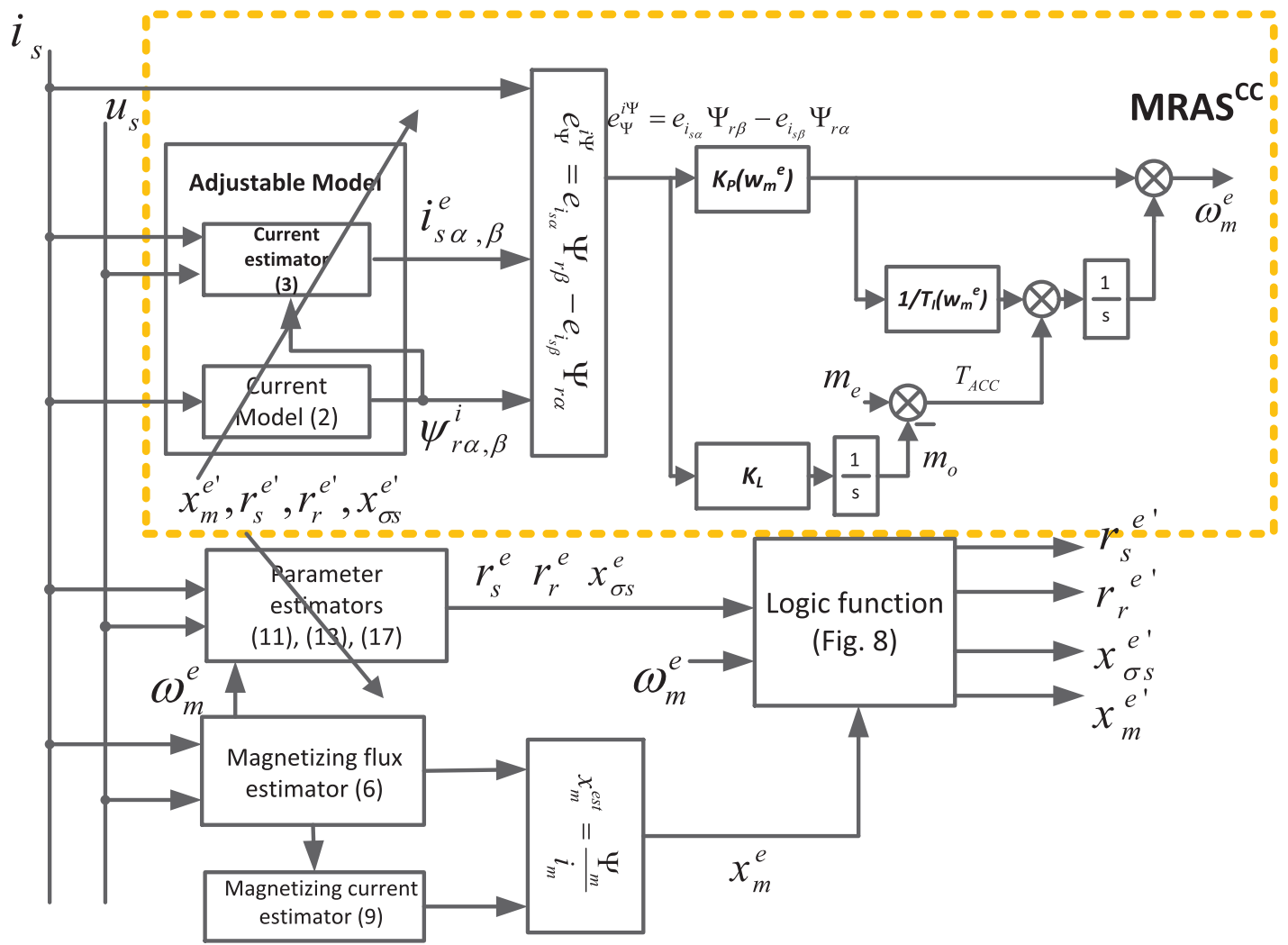

Fig. 7. Universal estimator scheme

Simultaneous operation of all parameter and speed estimators is impossible and can lead to system instability; therefore, it is necessary to sequentially start individual modules in such a way as to ensure the best possible system operation. To obtain this, the Logic function (from Fig. 7) is used. The idea is based on the sequential switching of the estimated parameters in the time function (Fig. 8). Parameter changes take place in a longlasting way; therefore, this way of operating individual estimators will not affect the quality of estimation in practical solutions.

In addition, it was assumed, that only magnetizing reactance estimator operates continuously.

On Fig 8, the periodic work of IM parameter estimators (Logic unction idea) is presented. 


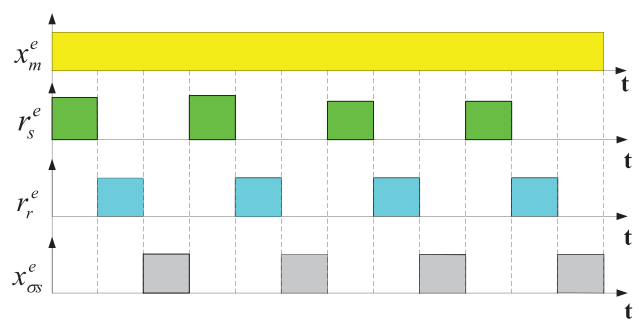

Fig. 8. Working times of individual IM parameter estimators

The parameter estimators should only work for steady speeds. During dynamic states (when $d \omega / d t \neq 0$ ), these mechanisms should be turned off and the parameters should take the last values determined for the steady state. This approach also affects in a significant way to improve the properties of the universal estimator.

Figure 9 shows the operation of the sequential estimation of the induction motor parameters for the assumed trajectories of their changes.
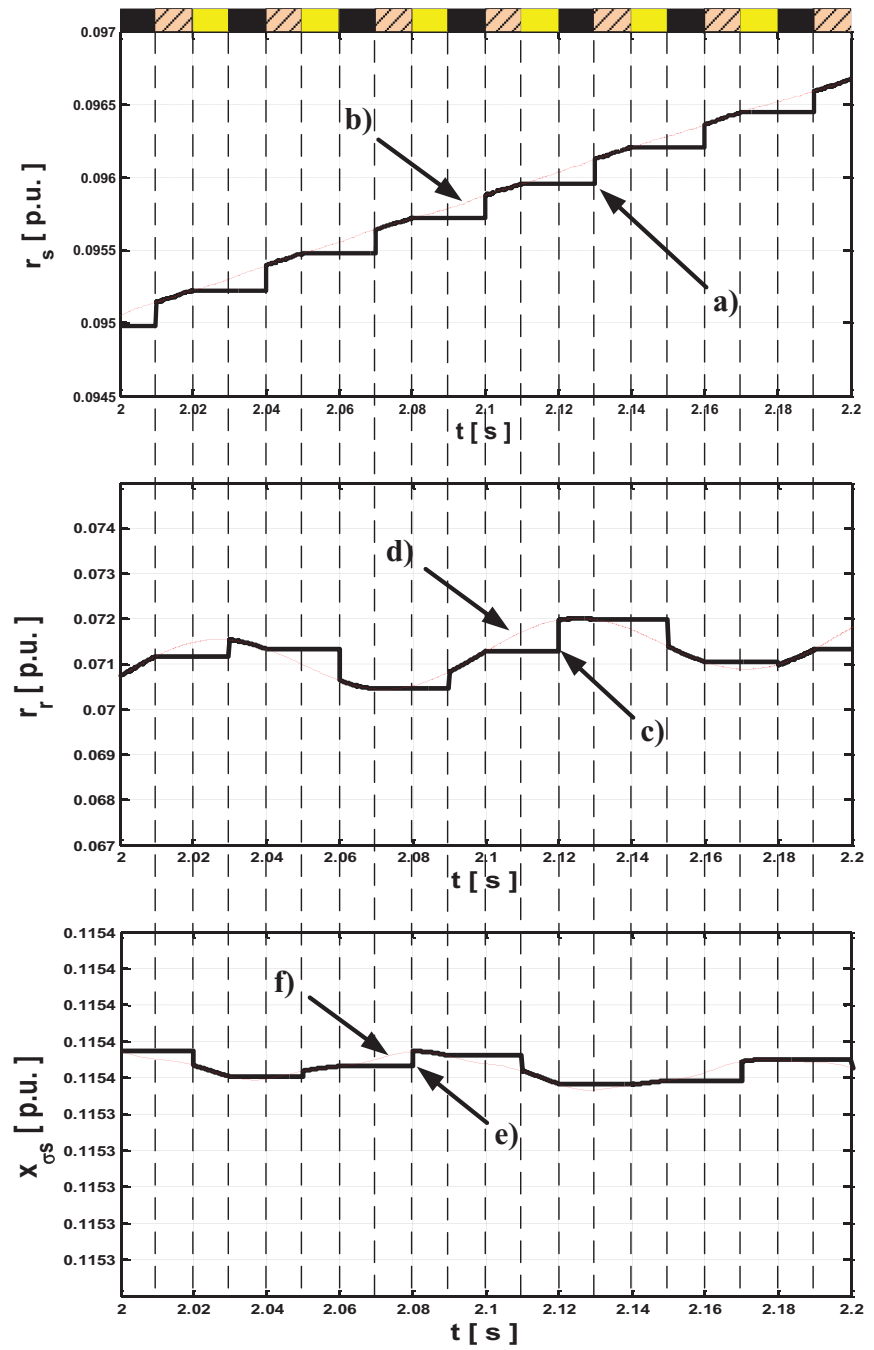

- operating period, in which only the rotor resistance estimator works,

$\measuredangle \triangle$ - operating period, in which only the stator resistance estimator works,

- operating period, in which only the stator leakage reactance estimator works,

Fig. 9. Estimated and sequential estimated stator resistances $(a, b)$, rotor resistance $(c, d)$, stator leakage reactance $(e, f)$ 
It is clearly visible, that at one moment, the output of only one estimator is active. This does not mean, however, that the mechanism of adaptation of individual parameters is switched off sequentially. This mechanism works in a continuous way, tracking the current values of individual parameters.

\subsection{Universal speed and flux estimator - Experimental tests}

Described universal estimator and control algorithms have been developed in a control computer that is based on a Power PC 750GX and TMS320F240 DSP DS1103 board. A DSP control board is installed in the extension box connected with the PC using fibre optics, which includes multi-channels of ADC, DAC, PIO and encoder interface circuits (Dybkowski and Orlowska-Kowalska, 2012). The speed signal acquisition has been performed with a 5000 imp./rev. incremental optical encoder, only for comparison with the estimated one. Induction motor was loaded using the second machine controlled by the inverter (torque control). The schematic diagram of the experimental test bench is shown in Fig. 10 (Dybkowski and Orlowska-Kowalska, 2012). The sampling time in the experimental test was equal $100 \mu \mathrm{s}$. The experimental set-up is composed of the $1.1 \mathrm{~kW}$ IM (fed from the voltage source inverter (VSI)) and a load machine (IM supplied from an AC inverter) (Fig. 10). The rated data of the tested IM are shown in the Appendix.

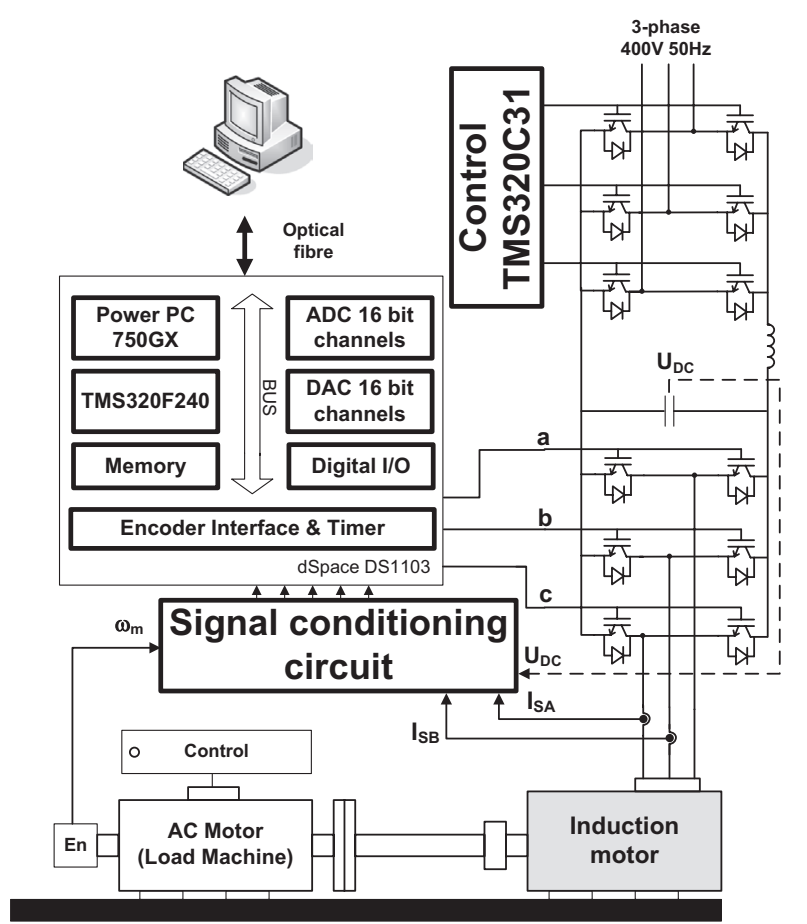

Fig. 10. Schematic diagram of the laboratory test bench (Dybkowski and Orlowska-Kowalska, 2012)

The proposed estimator was tested in the wide range of the rotor speed and load changes. The Fig. 11 to Fig. 15 show the waveforms of the selected state variables of the induction motor and the estimated IM parameters, stator resistance $\left(r_{s}\right)$, rotor resistance $\left(r_{r}\right)$, magnetizing reactance $\left(x_{m}\right)$ and stator leakage reactance $\left(x_{\sigma s}\right)$.

It is clearly visible that the systems estimate the IM parameters correctly in the states set for different rotor speed (Fig. 11-Fig. 15). To show in detail the value of the estimated $r_{r}$, the axis $Y$ in Fig. 11 was limited to the 0.1 [p. u.]. In Fig. 14 and Fig. 15, the transients with the peak are demonstrated.

IM parameters are not determined continuously but switched over by the supervisory system. During rotor speed changes, the estimated values are subjected to temporary oscillations, which, after stabilizing the speed and the flux at the level of the set points, stabilize.

In the low speed region, the stator resistance is estimated with relatively big error, so the estimation algorithm must be switch off in the low speed region (this problem is connected with the application of the voltage model in the estimators). The voltage model can reconstruct the rotor (or stator) flux with relatively big error in the low speed region - for low stator voltages (it is related to the voltage drop on the inverter). In experimental results, the limit was set to the $0.05 \omega_{m N^{*}}$ 

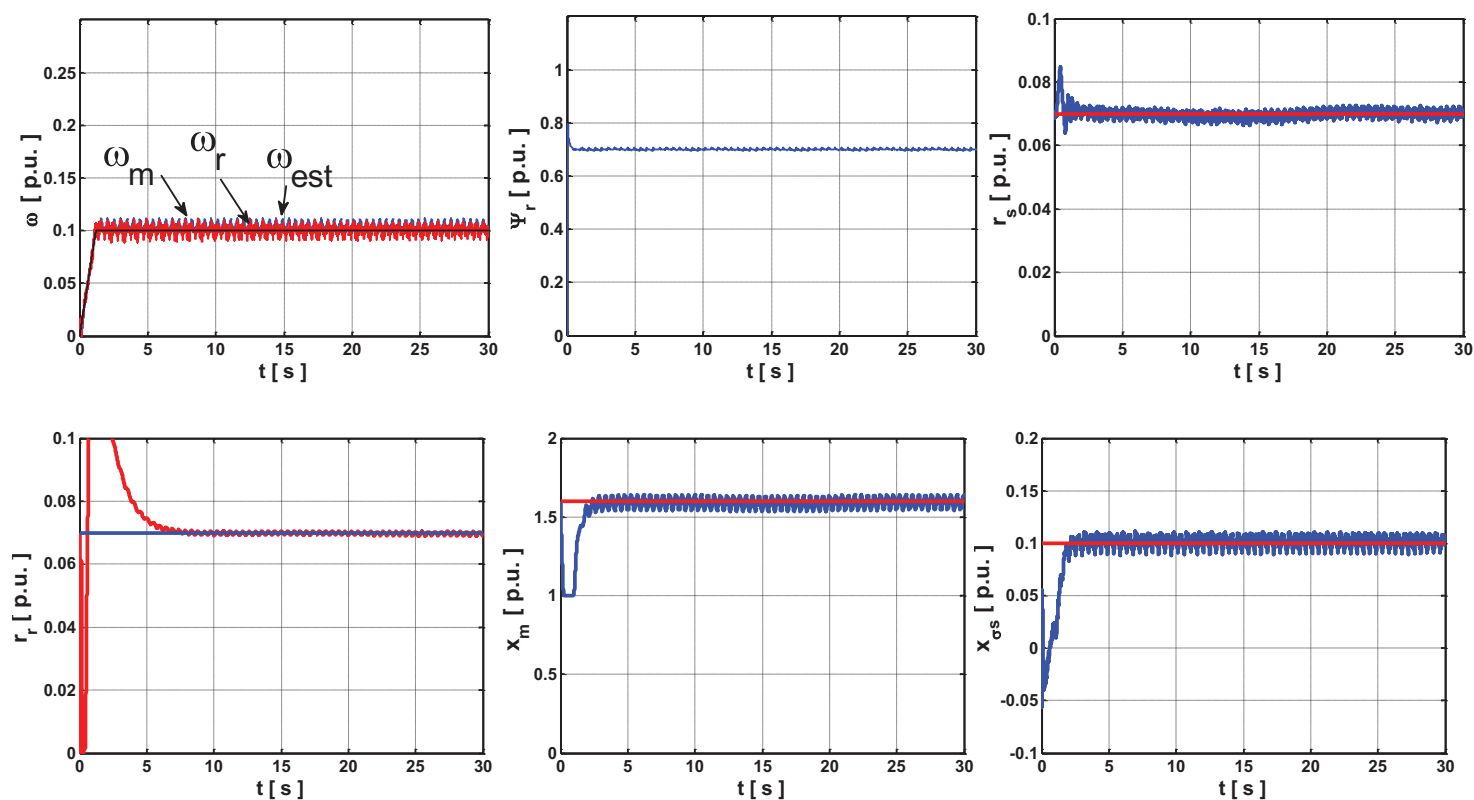

Fig. 11. Selected IM state variables and estimated parameters in the sensorless drive with a universal estimator $\omega_{m}=0.1 \omega_{m N^{*}} m_{\circ}=0.2 m_{o N}$
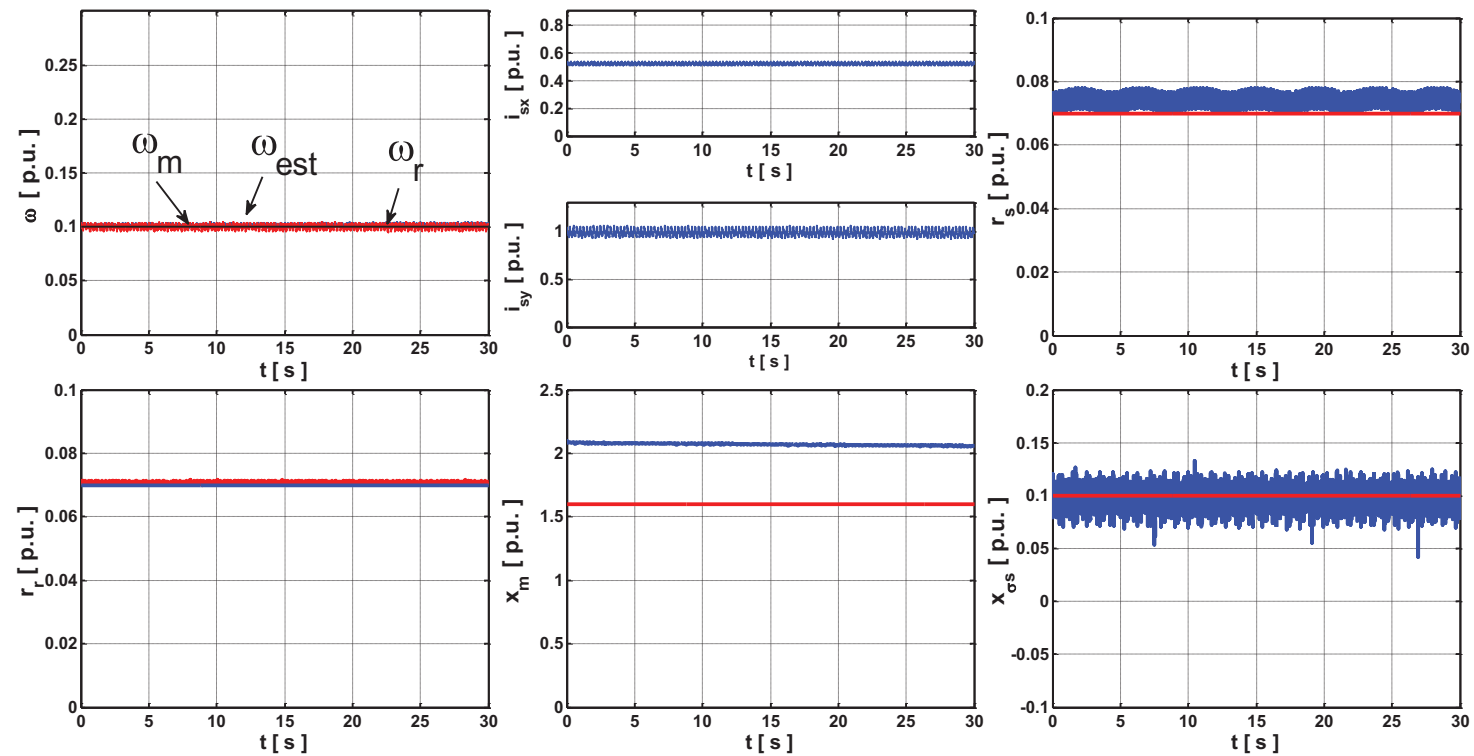

Fig. 12. Selected IM state variables and estimated parameters in the sensorless drive with a universal estimator $\omega_{m}=0.1 \omega_{m N^{\prime}} m_{0}=m_{o N}$

Magnetizing reactance estimator, similar to the stator resistant estimator, should be limited in the small speed region. During the start-up operation, all the estimated parameters must be set to the nominal (or calculated earlier) parameters.

Figure 12 shows waveforms of the sensorless drive operating at a speed of $\omega_{m}=0.1 \omega_{m N}$ and full load torque. The rotor flux is kept at the nominal value, the stator current components behave in a correct manner. The speed estimation error oscillates around zero. The estimated value of the magnetizing reactance is bigger than the value determined during the test in the stopped state because the drive is working under load. In this condition, this parameter increases because the magnetizing flux depends not only on the stator flux but also on the stator current, 
which increases during load torque. In this case, the value of the magnetizing reactance increases. As before, the IM parameters are estimated properly.
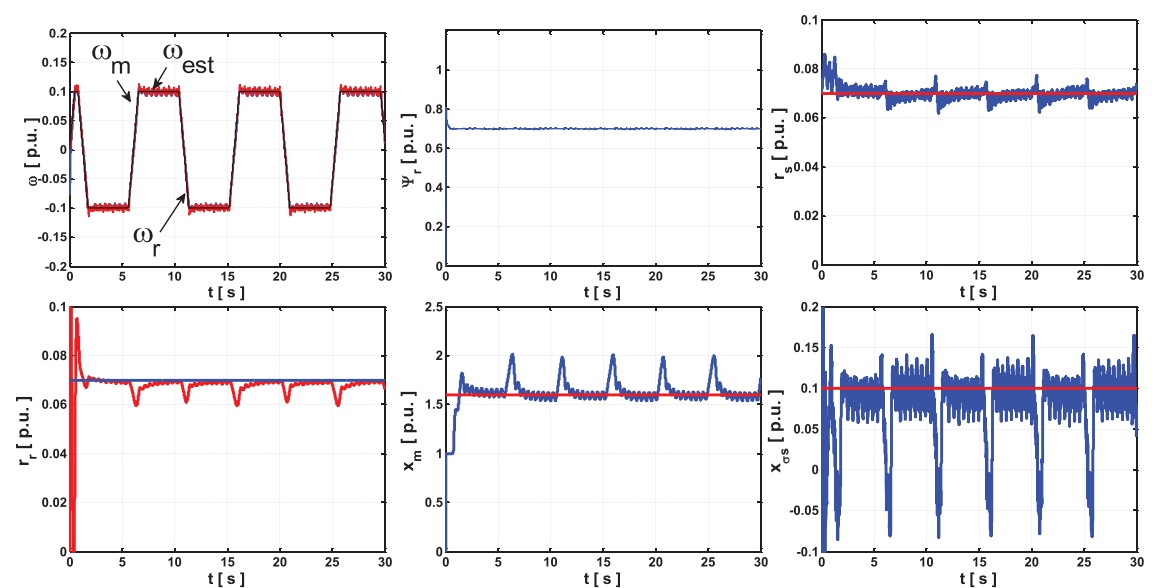

Fig. 13. Selected IM state variables and estimated parameters in the sensorless drive with a universal estimator $\omega_{m}= \pm 0.1 \omega_{m N}, m_{0}=0.2 m_{o N}$
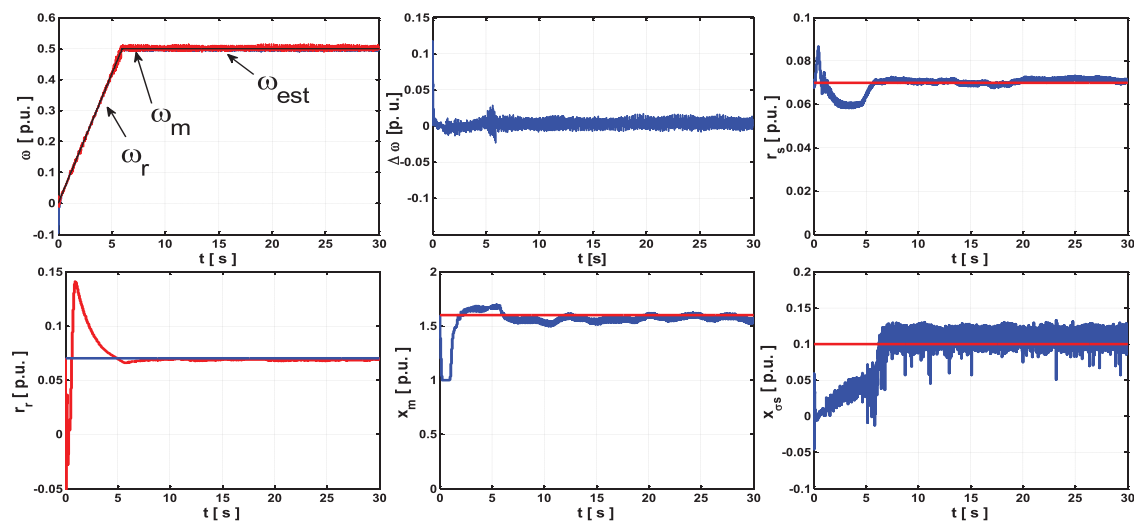

Fig. 14. Selected IM state variables and estimated parameters in the sensorless drive with a universal estimator $\omega_{m}=0.5 \omega_{m N^{\circ}} m_{0}=0.2 m_{o N}$

Drive was tested for higher speed as well(Fig.14), in the field weakening region and during the cyclic reverse operation (Fig. 13). Rotor flux is equal with the reference value. Parameters are estimated correctly. Only for dynamic states, the estimation errors are visible (on the parameters).
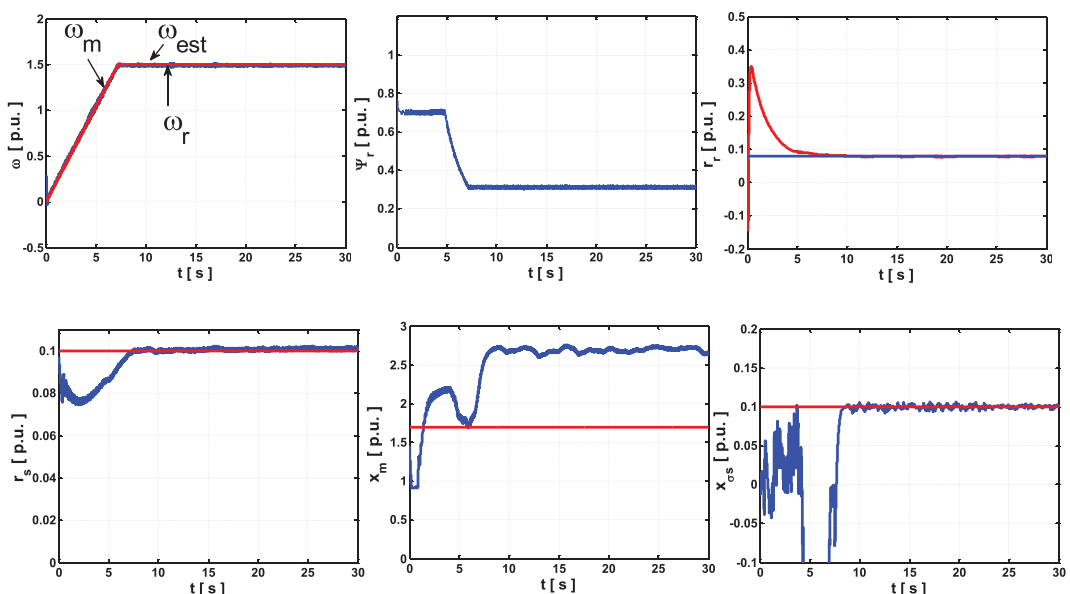

Fig. 15. Selected IM state variables and estimated parameters in the sensorless drive with a universal estimator $\omega_{m}=1.5 \omega_{m N}, m_{o}=0.2 m_{o N}$ 
Experimental tests were performed in a relatively low periodic of time and the temperature of the drive was constant (nominal). It is obvious that the rotor resistance depended on temperature and drive condition (Barut et al., 2012; Dybkowski, 2013; Proca and Keyhani, 2002). This influence was tested by the author in different papers. The proposed estimator can be used for the rotor faults detections (Dybkowski, 2013).

It can be unequivocally stated, that the estimators of parameters $\left(r_{r}, r_{s}\right.$ and $\left.x_{\sigma s}\right)$ must be turned off when $d \omega / d t \neq 0$, which has already been signalled.

It is visible that the estimated rotor resistance is different for different dynamical properties. If these states are long, the estimation process is not correct. It was shown that the universal estimator works properly in the sensorless induction motor drive systems. Experimental results (obtained in the laboratory set up with ds 1103 card) confirmed the theoretical and simulation results.

Simulation and experimental tests show that magnetizing reactance and stator resistance can be used simultaneously with MRAS type speed and flux estimator. Other parameters can be used as additional systems to improve the drive properties.

\section{Conclusions}

In the paper, the concept of the novel universal speed and flux estimator was proposed. System was extended by the additional induction motor parameter estimators. It was proved that all the estimators work correctly in wide speed changes. Magnetizing reactance and the stator resistance estimator should be used at the same time with the speed and flux estimator. Other estimators are additional systems, which can be used for drive working in the heavy conditions. The main problem, which was marked in the paper, is connected with the dynamical states. During this condition, the additional estimators estimate the parameters with relatively big errors and should be switched off during this condition.

\section{Appendix}

Motor rated data: $P_{N}=1.1[\mathrm{~kW}], \mathrm{U}_{N}=230 / 400[\mathrm{~V}], \mathrm{I}_{N}=5.0 / 2.9[\mathrm{~A}], n_{N}=1380[\mathrm{rpm}], f_{N}=50[\mathrm{~Hz}], p_{b}=2$

Parameters of the IM equivalent circuit: $R_{s}=5.9[\Omega], R_{r}=4.5[\Omega], X_{s}=131.1[\Omega], X_{r}=131.1[\Omega], X_{m}=123.3[\Omega]$

Per unit system calculation methodology (reference values): $U_{b}=\sqrt{2} U_{N f}, I_{b}=\sqrt{2} I_{N}, Z_{b}=U_{b} / I_{b}, \omega_{b}=2 \pi f_{N}$, $\Psi_{b}=U_{b} / \omega_{b}, S_{b}=(3 / 2) U_{b} I_{b}, M_{b}=S_{b} p_{b} / \omega_{b}$,

Parameters in per unit system: $r_{s}=R_{s} / Z_{b}, r_{r}=R_{r} / Z_{b}, x_{s}=1_{s}=X_{s} / Z_{b}, x_{r}=1_{r}=X_{r} / Z_{b}, x_{m}=I_{m}=X_{m} / Z_{b}$

\section{References}

Alonge, F., Cangemi, T., D'lppolito, F., Fagiolini, A. and Sferlazza, A. (2015). Convergence Analysis of Extended Kalman Filter for Sensorless Control of Induction Motor. IEEE Transactions on Industrial Electronics, 62(4), pp. 2341-2352.

Auger, F., Hilairet, M., Guerrero, J. M., Monmasson, E., Orlowska-Kowalska, T. and Katsura, S. (2013). Industrial Applications of the Kalman Filter: A Review. IEEE Transactions on Industrial Electronics, 60(12), pp. 5458-5471.

Barut, M. (2010). Bi-input-extended Kalman FilterBased Estimation Technique for Speed-Sensorless Control of Induction Motors. Energy Conversion and Management, 51(10), pp. 2032-2040.
Barut, M., Bogosyan, S. and Gokasan, M. (2008). Experimental Evaluation of Braided EKF for Sensorless Control of Induction Motors. IEEE Transactions on Industrial Electronics, 55(2), pp. 620-632.

Barut, M., Demir, R. and Inan, R. (2012). Real-Time Implementation of Bi Input-Extended Kalman FilterBased Estimator for Speed-Sensorless Control of Induction Motors. IEEE Transactions on Industrial Electronics, 59(2), pp. 4197-4206.

Dybkowski, M. (2013). Estimation of Speed in a Vector Controlled Induction Motor Drive - Selected Problems. Prace Naukowe Instytutu Maszyn, Napędów i Pomiarów Elektrycznych Politechniki 
Wrocławskiej nr 67. seria Monografie $\mathrm{nr} 20$. Wrocław (in Polish).

Dybkowski, M. and Orlowska-Kowalska, T. (2010). Speed sensorless induction motor drive with magnetizing reactance estimation. In: 2010 14th International Power Electronics and Motion Control Conference (EPE/PEMC), Ohrid, Macedonia, on CD, 2010.

Dybkowski, M. and Orlowska-Kowalska, T. (2012). Performance of the speed sensorless induction motor drive for traction application with MRAS type speed and flux estimator, In: 2012 13th International Conference on Optimization of Electrical and Electronic Equipment (OPTIM). Brasov, Romania: IEEE, pp. 477-481.

Dybkowski, M., Klimkowski, K. and Orlowska-Kowalska, T. (2014). Speed sensor fault tolerant direct torque control of induction motor drive. In: 2014 16th International Power Electronics and Motion Control Conference and Exposition (PEMC). Warna, Bulgaria: IEEE, 2014, pp. 679-684.

Holtz, J. (2006). Sensorless Control of Induction Machines-With or Without Signal Injection? IEEE Transactions on Industrial Electronics, 53(1), pp. 7-30.

Isermann, R. (2006). Fault Diagnosis Systems. An Introduction from Fault Detection to Fault Tolerance. New York: Springer.

Kubota, H., Matsuse, K. and Nakano, T. (1984). New control method of inverter - fed induction motor drive by using state observer with rotor resistance identification. In: IEEE/IAS Annual Meeting, Conference Record, pp. 601-606.

Levi, E., Sokola, M. and Vukosavic, S. N. (2000). AMethod for Magnetizing Curve Identification in Rotor Flux Oriented Induction Machines. IEEE Transactions on Energy Conversion, 15(2), pp. 157-162.

Orlowska-Kowalska, T. (2003). Sensorless Induction Motor Drives. Wroclaw, Poland: Wroclaw University of Technology Press.

Orlowska-Kowalska, T. and Dybkowski, M. (2010). Stator-Current-Based MRAS Estimator for A Wide
Range Speed-Sensorless Induction-Motor Drive. IEEE Transactions on Industrial Electronics, 57(4), pp. 1296-1308.

Orlowska-Kowalska, T., Tarchala, G. and Dybkowski, M. (2014). Sliding-Mode Direct Torque Control and Sliding-Mode Observer with A Magnetizing Reactance Estimator for the Field-Weakening of the Induction Motor Drive. Mathematics and Computers in Simulation, 98, pp. 31-45.

Proca, A. B. and Keyhani, A. (2002). Identification of Variable Frequency Induction Motor Models from Operating Data. IEEE Transactions on Energy Conversion, 17(1), pp. 24-31.

Rasmusen, H., Kundsten, M. and Tonnes, M. (1999). Parameter Estimation of Inverter and Motor Model at Standstill Using Measured Current Only. IEEE Transactions on Industrial Electronics, 46(1), pp. 139-149.

Schauder, C. (1992). Adaptive Speed Identification for Vector Control of Induction Motors without Rotational Transducers. IEEE Transactions on Industry Applications, IA-28(5), pp. 1054-1060.

Smith, A. N., Gadoue, S. M. and Finch, J. W. (2016). Improved Rotor Flux Estimation at Low Speeds for Torque MRAS-Based Sensorless Induction Motor Drives. IEEE Transactions on Energy Conversion, 31(1), pp. 270-282.

Tarchała, G., Dybkowski, M. and Orłowska-Kowalska, T. (2011). Analysis of the chosen speed and flux estimators for sensorless induction motor drive. In: Industrial Electronics (ISIE). 2011 IEEE International Symposium on, CD, Gdańsk.

Vasić, V., Vukosavic, N. and Levi, E. (2003). A Stator Resistance Estimation Scheme for Speed Sensorless Rotor Flux Oriented Induction Motor Drives. IEEE Transactions on Energy Conversion, 18(4), pp. 476-483.

Zorgani, Y. A., Koubaa, Y. and Boussak, M. (2010). Simultaneous Estimation of Speed and Rotor Resistance in Sensorless ISFOC Induction Motor Drive Based on MRAS Scheme. Roma: ICEM. 